\title{
Addendum
}

\section{Opioid analgesics: Is it time for risk stratification prior to use? - ADDENDUM}

Maureen A. Allen

doi: $10.1017 / \mathrm{cem} .2017 .424$

In the issued erratum notice ${ }^{1}$ for "Opioid analgesics: Is

REFERENCES

it time for risk stratification prior to use?," 2 the wrong DOI was cited for the original article.

The publisher regrets this error, and the notice has been updated.

1. Allen MA. Opioid analgesics: Is it time for risk stratification prior to use? - ERRATUM. CFEM 2017;epub, doi:10.1017/ cem.2017.424.

2. Allen MA. Opioid analgesics: Is it time for risk stratification prior to use? CFEM 2017;19(5):414. doi:10.1017/cem.2017.359. 